\section{PARP inhibitor approved, despite vote}

The US Food and Drug Administration approved AstraZeneca's first-in-class drug Lynparza (olaparib) for treating ovarian cancer linked to specific genetic mutations. Lynparza-a poly ADP-ribose polymerase (PARP) inhibitor-was given a green light on December 19 for treating ovarian cancer in women with mutated $B R C A 1$ and $B R C A 2$ genes, who have already failed on three or more chemotherapy treatments. The accelerated approval is a surprise considering an earlier negative panel vote (11-2) against recommending the drug. The agency approved the drug based on the drugmaker's reanalysis of trial data in patients with defective BRCA genes. The drug also won the go-ahead from the European Commission on the previous day. AstraZeneca projects $\$ 2$ billion a year sales for Lynparza, although the indication is narrow. It is also a win for Myriad Genetics, as Lynparza was approved in conjunction with the BRACAnalysis companion diagnostic test to identify patients with BRCA1/2 BRCA mutations. Under the FDA's accelerated-approval program, remaining on the market is contingent on verifying the drug's benefits in later studies.

\section{Biogen Idec bets on chronic pain}

Biogen Idec of Cambridge, Massachusetts, will expand its neuropathic pain portfolio with the purchase of Convergence Pharmaceuticals of Cambridge, UK. Biogen Idec will pay $\$ 200$ million upfront and up to $\$ 475$ million in milestone fees to Convergence, a developer of ion channel modulators for pain management that was spun out of GlaxoSmithKline. At the center of the deal is Convergence's experimental drug CVN1014802, currently in phase 2 testing as a treatment for trigeminal neuralgia, an orphan disease consisting of debilitating, episodic facial pain. CVN1014802 is a novel, small-molecule sodium-channel blocker that preferentially inhibits Nav 1.7 ion channels, a target implicated by genetics studies in human pain. The drug has also succeeded in a proof-ofconcept trial for sciatica and has potential for treating other neuropathic pain states.

\section{Merck buys BET family firm}

Swiss biotech OncoEthix's innovative, epigenetic, anti-cancer approach attracted a \$110-million upfront payment, and potentially another $\$ 265$ million in milestones from Merck in December. With the acquisition, the Kenilworth, New Jersey-based big pharma gains three first-in-class agents to a novel target or a novel mode of action, including OncoEthix's lead product OTX015, currently in phase 1b studies for hematological cancers and solid tumors. OTX015 is an oral small-molecule bromodomain inhibitor, from the BET (bromodomain and extraterminal domain) family of proteins, specifically targeting BET 2/3/4. Bromodomains are key motifs in protein-histone associations and play critical roles in the transcription of key growth control. Dysfunctions of bromodomain proteins have been linked to various forms of cancer. OTX015 was in-licensed from Mitsubishi Tanabe Pharma in 2012.
AC Immune, of Lausanne, Switzerland, and the S. San Francisco, California-based Genentech arm of Roche also detected a trend toward a cognitive benefit in a patient subgroup with mild disease in a phase 2 trial of crenezumab that failed to meet its primary endpoints. At the time of writing, Genentech had not yet decided on whether it would continue the program. "We are confident Roche will take the right decision. The data are very promising. Everyone is convinced of that," says AC Immune CEO Andrea Pfeifer. Biogen Idec's anti-amyloid antibody aducanumab, which it in-licensed from Neurimmune, of Schlieren, Switzerland, had time- and dose-dependent effects on $A \beta$ reduction in patients' brains, as well as a benefit in cognition. "To date, ours is the first observation of beta amyloid removal correlated with improved cognition," says Doug Williams, executive vice president of R\&D at Biogen Idec, of Cambridge, Massachusetts. The company plans to report the full dataset at the Alzheimer's and Parkinson's Disease Congress in Nice, France, in March.

The case for any of these antibodies is still far from proven, however. "We should be wary of overinterpreting beneficial effects that may occur as a result of anti- $\mathrm{A} \beta$ treatments before jumping to conclusions. So far, we have not seen data to support a robust positive effect of anti-A $\beta$ treatments in any trial," Bryce Vissel and Gary Morris, of the Garvan Institute of Medical Research, in Sydney, Australia, state by email.

Small-molecule inhibitors of APP processing work upstream from anti-amyloid antibodies by targeting the enzymes that cleave APP into $A \beta$ and other amyloid species. They offer the theoretical advantage of shutting down the production of $\mathrm{A} \beta$ before aggregation can occur.

BACE is currently industry's favored target following Lilly's unsuccessful phase 3 trial of semagacestat, a $\gamma$-secretase inhibitor, which actually had negative effects on patients' cognition and functioning. It also raised the incidence of nonmelanoma skin cancers in patients, due to its inhibition of Notch signaling. The $\gamma$-secretase enzyme has dozens of substrates, which makes it difficult to target cleanly. "For BACE there are a couple of other proteins but it doesn't seem to be nearly as problematic from the standpoint of having multiple substrates," Siemers says. Bart De Strooper, of the Flanders Institute for Biotechnology, in Leuven, Belgium, disagrees. "Basically it's a lack of knowledge that allows us to think this," he says. "I'm a bit worried we'll end up as we did with the semagacestat trial." New BACE substrates continue to be discovered, and the optimal level of inhibition of the target has not been defined.
De Strooper is also critical of the general rush to move investigational agents directly into large-scale phase 3 trials based on minimal clinical data. "We should be very careful with the few drug targets that we have," he says. "Once you have a phase 3 failure, the drug is completely burnt." In that context, he argues that Lilly's phase 3 trial of semagacestat has had a disproportionately negative effect on research into the role of $\gamma$-secretase in Alzheimer's. The adverse effects seen in the study may have arisen because of the specific dosing regimen adopted, he suggests in a recent commentary (Cell 159, 721-726, 2014). Partial but continuous inhibition of the target, instead of the schedule Lilly employed, which involved intermittent, complete inhibition of the target, could, he says, have mitigated the effects on Notch signaling, which follows an oscillatory pattern. Companies should, he says, invest more time on exploratory phase 2 trials, to work out the finer points of a drug's mechanism and to identify appropriate patient populations, rather than staking everything on one large study.

Whether any company has made a winning bet will become apparent in the next several years, as data from the current batch of largescale trials start to flow. A better understanding of the underlying biology is developing in parallel. "We suggest that hope for progress will most likely come from innovative thinking that integrates current data regarding amyloid with emerging advances in neuroscience, to create a newer more holistic view of AD," Vissel and Morris note. More careful exploration of what is already available could also bear fruit. "I think we already have drugs that could help in certain subgroups or if dosed at the right moment," De Strooper says.

\section{Cormac Sheridan Dublin}

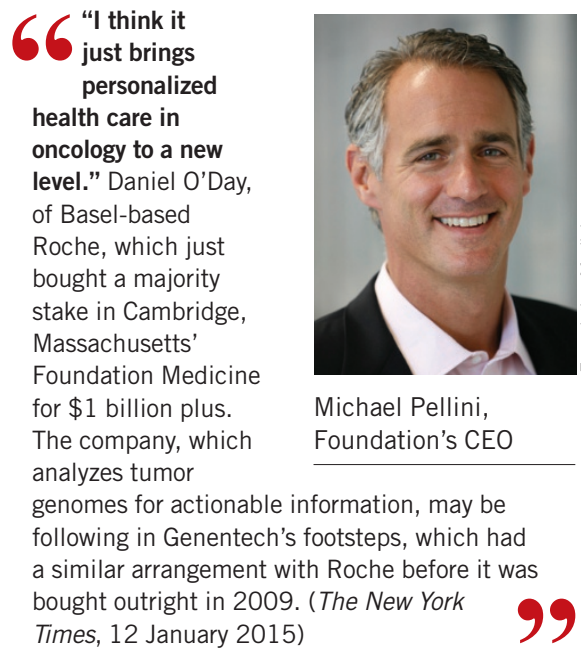

